\author{
A. Meyer ${ }^{1}$, W. Pfeilschifter ${ }^{1,2}$, C. Polkowski ${ }^{3}$ \\ ${ }^{1}$ Klinik für Neurologie, Universitätsklinik Frankfurt, Frankfurt am Main, Deutschland \\ ${ }^{2}$ Klinik für Neurologie und Klinische Neurophysiologie, Städtisches Klinikum Lüneburg, Lüneburg, Deutschland \\ ${ }^{3}$ Institut für Neuroradiologie, Universitätsklinik Frankfurt, Frankfurt am Main, Deutschland
}

\title{
Luftnot und Kauderwelsch
}

\begin{abstract}
Anamnese
Eine 39-jährige Patientin stellte sich in einer peripheren, internistischen Notaufnahme aufgrund von Luftnot, einem Schwellungsgefühl des rechten Arms und Geschmacksverlust vor. Etwa 1 Woche vor der Vorstellung bestanden über mehrere Tage andauernde Halsschmerzen, Fieber sei jedoch nie aufgetreten. An Vorerkrankungen seien ein Zentralarterienverschluss des rechten Auges ca. 9 Monate zuvor und eine dabei erstdiagnostizierte Faktor-V-Leiden-Mutation bekannt.
\end{abstract}

\section{Internistische Aufnahme}

Die internistische Untersuchung ergab lediglich Petechien an beiden Unterarmen und beiden Beinen. Eine objektivierbare Schwellung oder Rötung des rechten Armes lag nicht vor, hier bestanden auch keine Schmerzen. Es konnten eine Tachykardie (121 Schläge/min) und eine Tachypnoe (18/min) festgestellt werden. Die Sauerstoffsättigung unter Raumluft lag bei $99 \%$. Der SARS-CoV-2-PCR-Test verblieb negativ. Im Erstkontakt und nochmals während der Untersuchungen trat eine zunächst wegen türkischer Herkunft als Sprachbarriere gedeutete, transiente Sprachstörung auf. So befolgte die Patientin anfangs basale Aufforderungen, redete jedoch nur in unverständlichem Kauderwelsch. Während dieser Episoden bestand eine Monoparese des rechten Armes. Nach etwa $1 \mathrm{~h}$ sprach sie dann akzentfrei Deutsch und war allseits orientiert. Die Patientin selbst gab an, alle Gesprächsinhalte verstanden zu haben, aber nicht in der Lage gewesen zu sein, deutsche oder türkische Sprache zu produzieren. Eine Amnesie für die Episode bestand nicht. Iktale Stigmata im Sinne von Zungen- oder Wangenbiss, Enuresis, Enkopresis oder nestelnde Bewegungen wurden nicht beobachtet. $\mathrm{Zu}$ keiner Zeit traten Kopfschmerzen, Übelkeit oder Erbrechen auf.

Laborchemisch zeigten sich als auffällig eine hämolytische Anämie (Hb 6,4g/dl, Gesamtbilirubin 2,0 mg/dl, LDH $1248 \mathrm{U} / \mathrm{l})$, eine ausgeprägte Thrombozytopenie (8/nl) und eine Fibrinolyse (D-Dimere $2193 \mathrm{ng} / \mathrm{ml}$ ). Direkter und indirekter Coombs-Test verblieben negativ.

\section{Redaktion}

J. Bösel, Kassel

S. Schönenberger, Heidelberg

DGNeurologie $2021 \cdot 4(6): 452-456$

https://doi.org/10.1007/s42451-021-00376-w

Angenommen: 26. August 2021

Online publiziert: 22 . September 2021

(c) Springer Medizin Verlag GmbH, ein Teil von Springer Nature 2021

\section{Neurologische Befunde}

Bei Ankunft in der neurologischen Notaufnahme war die Patientin fokal-neurologisch unauffällig. Wegen des akuten Einsetzens und der bereits vollständigen Regredienz der neurologischen Symptomatik wurde unter dem Verdacht einer transitorisch-ischämischen Attacke zunächst eine zerebrale MRT (cMRT) durchgeführt, welche eine punktförmige Diffusionsrestriktion im Caput nuclei caudati rechts ergab. Diese war in der FLAIR-Sequenz (FLAIR: „fluid-attenuated inversion recovery") bereits vollständig demarkiert (Abb. 1). Da die Symptomatik durch diesen kleinen Infarkt nicht zu erklären war und monomorph auftrat, wurde unter der Annahme einer iktalen Genese kurzzeitig eine antikonvulsive Medikation mit Levetiracetam begonnen.

\section{Verdachtsgeleitete Nachanamnese}

Aufgrund der Laborbefunde und der ischämischen Endorganschäden bestand der Verdacht einer thrombotischen Mikroangiopathie. Die Patientin wies weder Fieber noch erhöhte Infektparameter (Differentialdiagnose [DD]: Sepsis) auf, eine Schwangerschaft (DD: Präeklampsie, HELLP-Syndrom) bestand ebenso wenig wie eine maligne oder rheumatologische Grunderkrankung. Anamnestisch hatte es weder eine Impfung (DD: „vaccine induced thrombotic thrombocytopenia“ [VITT]) noch eine Medikation mit Heparin (DD: heparininduzierte Thrombozytopenie [HIT]) oder eine Stammzelltransplantation gegeben. Es bestand keine Dauermedikation, und die Drogenanamnese war leer. Ein kürzlicher Infekt wurde ebenso wie blutige Diarrhöen von der Patientin verneint (DD: Shigatoxin induziertes hämolytisch-urämisches Syndrom [typisches HUS]). Die Familienanamnese war leer für thrombembolische Ereignisse oder unerklärte Tode <50 Jahren (DD: hereditäre thrombotische Mikroangiopathien).

\section{Diagnose, Therapie und Verlauf}

Nach Ausschluss dieser Differenzialdiagnosen erschien bei einem PLASMIC-Score (Tab. 1) von 7 Punkten somit eine thrombotisch-thrombozytopenische Purpura wahrscheinlich. Es wurden umgehend ADAMTS-13-Aktivität und Anti-ADAMTS-13-Inhibitoren bestimmt und probatorisch eine notfällige Plasmaseparation am selben Tag begonnen. Unterstützend erhielt die Patientin $100 \mathrm{mg}$ Prednisolon/Tag und für die ersten 3 Tage der Plasmapherese bis zur Normalisierung der Thrombozytopenie zusätzlich Caplacizumab.

Diagnose. Thrombotisch-thrombozytopenische Purpura

Die ADAMTS-13-Aktivität lag bei $0,21 \%$ (Referenz: 40-130\%), und es konnten Anti-ADAMTS-13-Antikörper 
Hier steht eine Anzeige.

Springer 

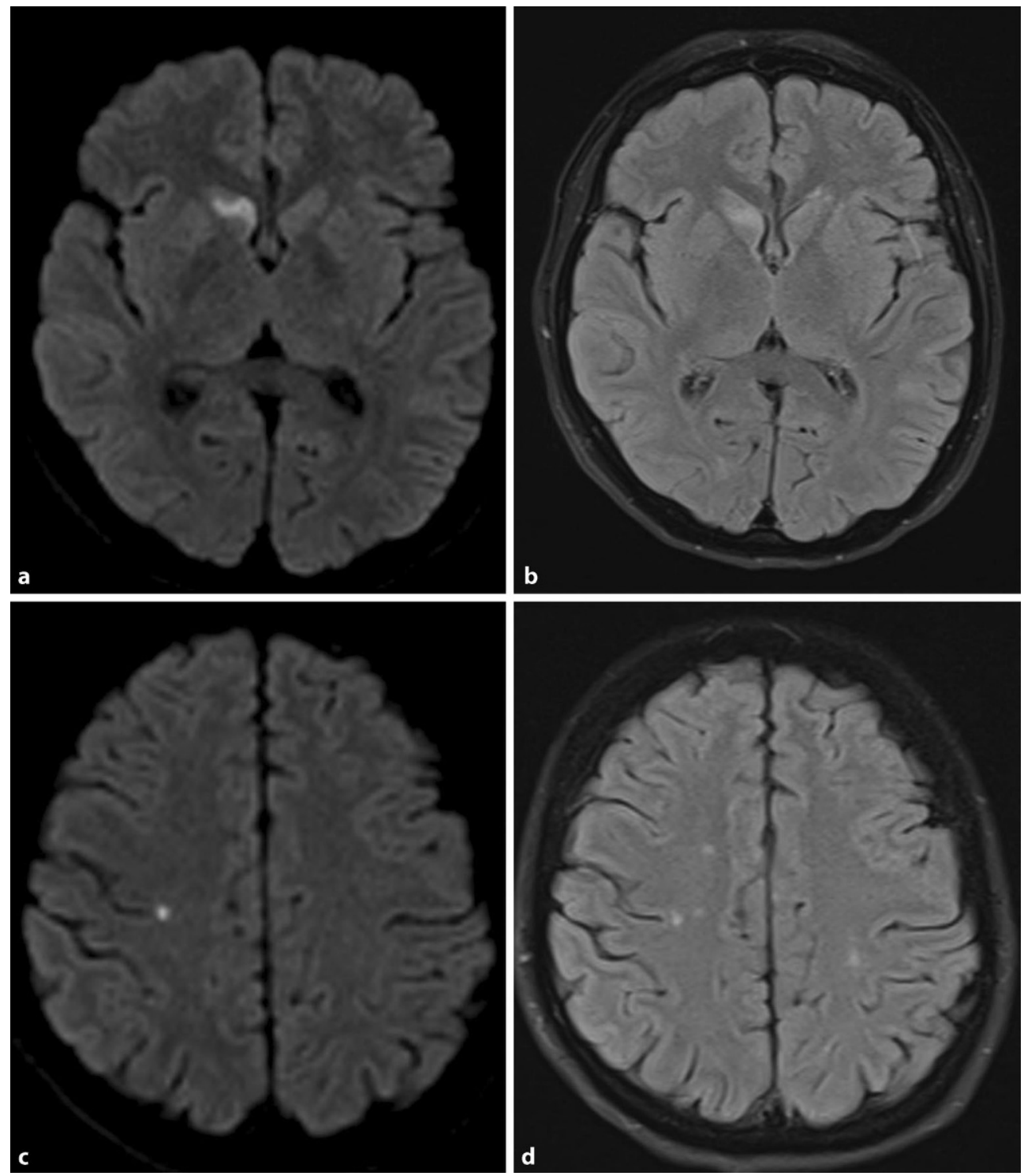

Abb. 1 Transversale Schnittbilder der zerebralen MRT im Verlauf. a,c DWI-Sequenzen (DWI: "diffusion weighted imaging"), b, d FLAIR-Sequenzen (FLAIR: „fluid-attenuated inversion recovery"), a Diffusionsrestriktion im Caput nuclei caudati rechts mit in $\mathbf{b}$ zu diesem Zeitpunkt bereits in FLAIR-demarkiertem Infarkt, c gegenüber der zerebralen MRT bei Aufnahme neue Diffusionsrestriktion im Stromgebiet der A. cerebri media rechts mit in $\mathbf{d}$ ebenfalls bereits FLAIR-demarkiertem Infarkt. (Die Bilder wurden freundlicherweise vom Institut für Diagnostische und Interventionelle Radiologie des Universitätsklinikums Frankfurt zur Verfügung gestellt)

Tab. 1 PLASMIC-Score zur Prädiktion einer thrombotischen Mikroangiopathie als Folge einer verminderten ADAMTS-13-Aktivität. (Mod. nach [3])

\begin{tabular}{|l|l|}
\hline PLASMIC-Score für die TTP & Punkte \\
\hline$<30$ Thrombozyten/nl & 1 \\
\hline $\begin{array}{l}\text { Hämolyse (indirektes Bilirubin }>2 \mathrm{mg} / \mathrm{dl} \text {, unkorrigierter Retiku- } \\
\text { lozytenanteil }>2,5 \% \text {, Haptoglobin unter der Nachweisgrenze) }\end{array}$ & 1 \\
\hline $\begin{array}{l}\text { Keine aktive Tumorerkrankung in den letzten 12 Monaten } \\
\text { Keine vorangegangene Stammzell- oder Organtransplantation }\end{array}$ & 1 \\
\hline "Mean corpuscular volume" (MCV) $<90$ fl & 1 \\
\hline "International normalized ratio" (INR) $<1,5$ & 1 \\
\hline Kreatinin im Serum $<2 \mathrm{mg} / \mathrm{dl}$ & 1 \\
\hline
\end{tabular}

0-4 Punkte entsprechen einem 0- bis 4\%igen Risiko einer TTP, 6-7 Punkte einem $>80 \%$ igen Risiko einer TTP nachgewiesen werden, sodass die Diagnose einer erworbenen thrombotisch-thrombozytopenischen Purpura gestellt werden konnte. Unter der angegebenen Therapie normalisierte sich die Thrombozytenzahl innerhalb von 4 Tagen (Abb. 2). Während der Therapie kam es zu transientem Verschwommensehen im linken oberen Gesichtsfeld und einer transienten Monoparese mit Kribbelparästhesie des linken Arms. Die daraufhin durchgeführte cMRT zeigte neue Infarkte im Praecuneus links und Gyrus postcentralis rechts (Abb. 1). Die Plasmaseparation wurde über insgesamt 8 Sitzungen durchgeführt. In den monatlichen Kontrollen seit der Entlassung klagte die Patientin nicht wieder über typische Beschwerden oder neurologische Ausfälle, und es konnten keine Endorganschäden festgestellt werden. Die Thrombozyten blieben immer grenzwertig niedrig, die ADAMTS-13-Aktivität normalisierte sich innerhalb von 6 Monaten. 


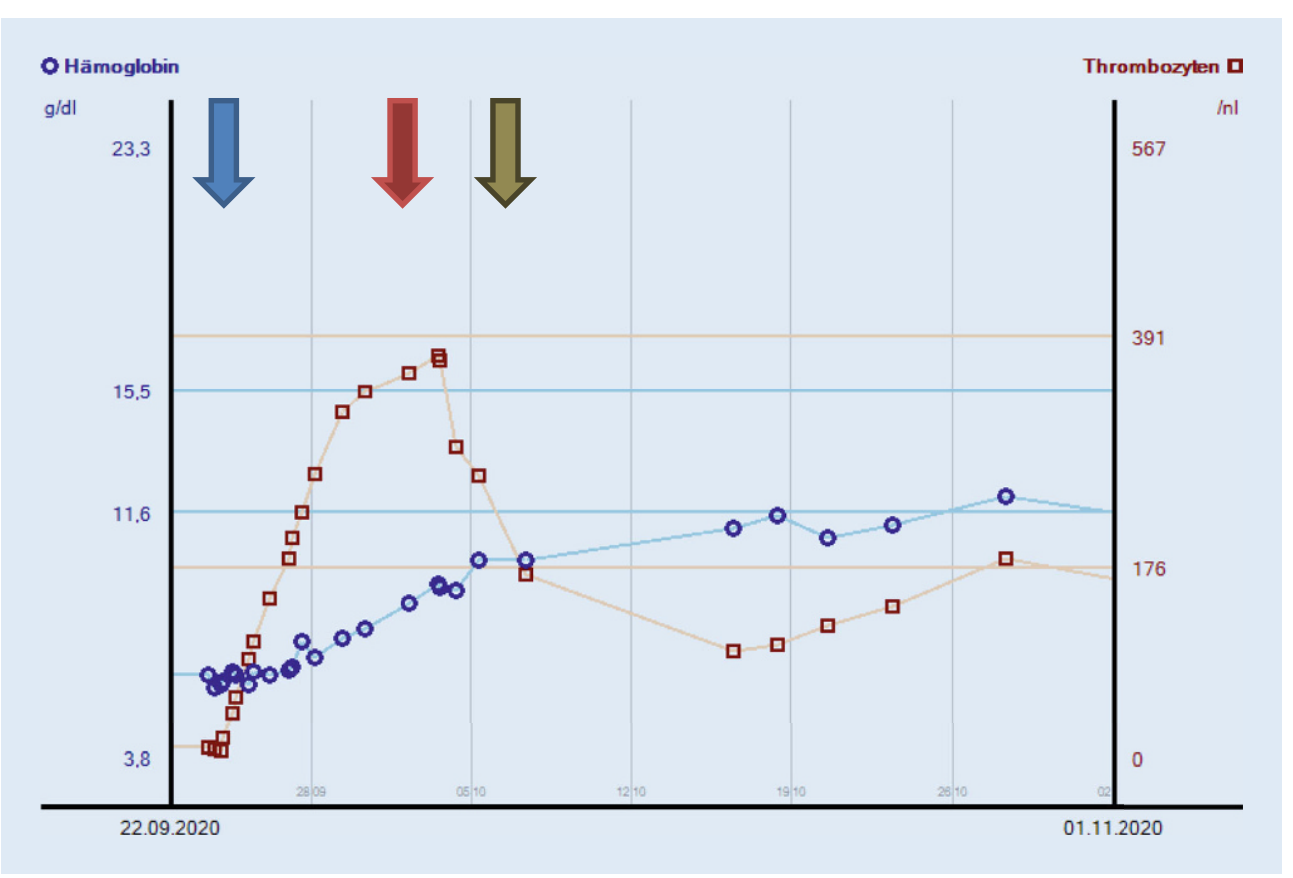

\author{
Abb. 2 Verlauf der Hämo- \\ globinkonzentration und \\ Thrombozytenzahl, blauer Pfeil \\ Tag der Aufnahme, roter Pfeil \\ Ende von Plasmaseparation \\ und Caplacizumab, grüner Pfeil \\ Entlassung aus dem Krankenhaus
}

\section{Diskussion}

Die erworbene, thrombotisch-thrombozytopenische Purpura (aTTP) gehört zur ätiopathogenetisch heterogenen Gruppe der thrombotischen Mikroangiopathien. Bei diesen Erkrankungen verursachen Thrombosen der kleinsten Gefäße Mikrozirkulationsstörungen und führen so zu Endorganschäden bis hin zum Multiorganversagen [1]. Der thrombotisch-thrombozytopenischen Purpura liegt dabei eine verminderte Aktivität der Protease ADAMTS-13 zugrunde, deren Aufgabe die Spaltung der endothelständigen Von-Willebrand-Faktor-Polymere ist. Ohne diese Proteolyse kommt es zur intravasalen Aktivierung der primären Hämostase, in deren Folge Thrombosen entstehen. Unterschieden werden bei der thrombotisch-thrombozytopenischen Purpura eine hereditäre Form mit reduzierter ADAMTS-13-Expression und eine erworbene Form mit neutralisierenden Antikörpern gegen ADAMTS-13 und dadurch reduzierter ADAMTS-13-Aktivität [2].

Klassischerweise wird der aTTP die Pentade aus Fieber (selten), neurologischen Symptomen, Thrombozytopenie, hämolytischer Anämie und Nierenfunktionsstörung (seltener als bei anderen thrombotischen Mikroangiopathien) zugeschrieben. Die Symptomatik ist jedoch vielfältig und abhängig von der Lokalisation der Mikrothromben, was eine frühe Diagnose häufig erschwert. Wegweisend sind die Laborbefunde mit Thrombozytopenie, hämolytischer Anämie und Nachweis von Fragmentozyten sowie die spezifischen Parameter ADAMTS13, ADAMTS-13-Aktivität und Anti-ADAMTS-13-Antikörper [2]. Für den klinischen Alltag steht mit dem PLASMIC-Score ein validiertes Screeningtool zur Verfügung (Tab. 1; [3]). Unbehandelt geht die aTTP mit einer Letalität von bis zu $70 \%$ einher [4]. Frauen sind häufiger betroffen als Männer, und die erste Episode tritt meistens zwischen dem 35. und 45. Lebensjahr auf. Die Rezidivraten sind unabhängig von der ADAMTS13Aktivität und liegen zwischen 10 und $40 \%$ innerhalb von 20 Jahren [5]. Die angegebenen Laborparameter eignen sich nicht zur Verlaufskontrolle. Hierfür sollten vielmehr regelmäBige klinische Kontrollen stattfinden. Eine Rezidivprophylaxe wird nicht durchgeführt.

Die Therapie sollte schon bei hochgradigem Verdacht schnellstmöglich und noch vor Eingang der spezifischen Laborparameter begonnen werden. Steht noch tagesgleich eine Plasmapheresetherapie zur Verfügung, ist diese Mittel der ersten Wahl zur Elimination der neutralisierenden Antikörper [4]. Der relevante Verlaufsparameter für die Therapiedauer ist hierbei die Thrombozytenzahl. Zusätzlich und bei fehlenden Plasmapheresekapazitäten wird Prednisolon zur Immunmodulation eingesetzt. Mit diesen Therapien konnte die Letalität auf $20-40 \%$ reduziert werden [5]. Bei fehlendem Therapieansprechen wird Rituximab empfohlen, obwohl hierfür keine Zulassung vorliegt [6]. Die Transfusion von Thrombozytenkonzentraten sollte nur erfolgen, wenn keine Plasmaseparation zur Verfügung steht [4].

Neu in der Therapie der aTTP ist Caplacizumab, ein humanisierter Antikörper, der die Bindung von Thrombozyten an den Von-Willebrand-Faktor verhindert. Damit ist Caplacizumab besonders in der Initialphase der Erkrankung bei noch reduzierter ADAMTS-13-Aktivität und damit vorhandenen Von-Willebrand-Faktor-Polymeren eine sinnvolle Ergänzung zur Vermeidung von Mikrothromben [7].

\section{Fazit für die Praxis}

- Bei jungen PatientInnen mit zentralen, fokal-neurologischen Symptomen sollte eine Blutentnahme mit Blutbild, LDH, Gerinnungsparametern und Nierenfunktionsparametern erfolgen.

- Eine Thrombozytopenie und eine hämolytische Anämie sollten an eine thrombotisch-thrombozytopenische Purpura denken lassen.

- Zur Abschätzung der Wahrscheinlichkeit ist der PLASMICScore ein gut validiertes Screeninginstrument. 
- Mit ADAMTS-13, der ADAMT13-Aktivität und dem Nachweis von ADAMTS-13-Inhibitoren können eine aTTP diagnostiziert und die autoimmune von der hereditären Genese differenziert werden.

- Schon bei klinischem Verdacht und passenden Standardlaborparametern sollte eine notfällige Plasmapherese initiiert werden.

- Insbesondere anfangs sollten Prednisolon und Caplacizumab die Plasmapheresetherapie ergänzen.

- Thrombozytentransfusionen sollten nur erfolgen, wenn eine Plasmaseparation nicht am selben Tag möglich ist.

- Rezidive sind häufig und können zunächst mit unspezifischen Symptomen einhergehen, ein guter, labortechnischer Verlaufsparameter steht nicht zur Verfügung.

Literatur

1. George JN, Nester CM (2014) Syndromes of thrombotic microangiopathy. N Engl J Med 371(7):654-666. https://doi.org/10.1056/NEJMra1312353

2. Tsai HM, Lian EC (1998) Antibodies to von Willebrand factor-cleaving protease in acute thrombotic thrombocytopenic purpura. N Engl J Med 339(22):1585-1594. https://doi.org/10.1056/NEJM199811263392203

3. Bendapudi PK, Hurwitz S, Fry A, Marques MB, Waldo SW, Li A, Sun L, Upadhyay V, Hamdan A, Brunner AM, Gansner JM, Viswanathan S, Kaufman RM, Uhl L, Stowell CP, Dzik WH, Makar RS (2017) Derivation and external validation of the PLASMIC score for rapid assessment of adults with thrombotic microangiopathies: a cohort study. Lancet Haematol 4(4):e157-e164. https://doi.org/10.1016/S23523026(17)30026-1

4. Rock GA, Shumak KH, Buskard NA, Blanchette VS, Kelton JG, Nair RC, Spasoff RA (1991) Comparison of plasma exchange with plasma infusion in the treatment of thrombotic thrombocytopenic purpura. Canadian Apheresis Study Group. N Engl J Med 325(6):393-397. https://doi.org/10.1056/NEJM199108083250604

5. Kremer Hovinga JA, Vesely SK, Terrell DR, Lämmle B, George JN (2010) Survival and relapse in patients with thrombotic thrombocytopenic purpura. Blood 115(8):1500-1511. https://doi.org/10.1182/blood-2009-09-243790(quiz 1662)

6. Zheng XL, Vesely SK, Cataland SR, Coppo P, Geldziler B, lorio A, Matsumoto M, Mustafa RA, Pai M, Rock G, Russell L, Tarawneh R, Valdes J, Peyvandi F (2020) ISTH guidelines for the diagnosis of thrombotic thrombocytopenic purpura. J Thromb Haemost 18(10):2486-2495. https://doi.org/10.1111/jth.15006(Erratum in: J Thromb Haemost 19(5):1381 (2021))

7. Scully M, Cataland SR, Peyvandi F, Coppo P, Knöbl P, Kremer Hovinga JA, Metjian A, de la Rubia J, Pavenski K, Callewaert F, Biswas D, De Winter H, Zeldin RK (2019) Caplacizumab treatment for acquired thrombotic thrombocytopenic purpura. N Engl J Med 380(4):335-346. https://doi.org/10.1056/NEJMoa1806311

\section{Korrespondenzadresse}

\section{Anjela Meyer}

Klinik für Neurologie

Universitätsklinik Frankfurt

Schleusenweg 2-16, 60528 Frankfurt am Main,

Deutschland

Anjela.meyer@kgu.de
Für diesen Beitrag wurden von den Autoren keine Studien an Menschen oder Tieren durchgeführt. Für die aufgeführten Studien gelten die jeweils dort angegebenen ethischen Richtlinien. Für Bildmaterial oder anderweitige Angaben innerhalb des Manuskripts, über die Patienten zu identifizieren sind, liegt von ihnen und/oder ihren gesetzlichen Vertretern eine schriftliche Einwilligung vor.

\section{Einhaltung ethischer Richtlinien}

Interessenkonflikt. A. Meyer, W. Pfeilschifter und C. Polkowski geben an, dass kein Interessenkonflikt besteht. 